\title{
Is the Primary Aetiology of Hypertension Unknown? Novel Views on Previous Assumptions
}

\author{
Andras Sikter $^{1 *}$ and Christian Sonne ${ }^{2}$ \\ ${ }^{1}$ Department of Internal Medicine, Municipal Clinic of Szentendre, Hungary \\ ${ }^{2}$ Department of Bioscience, Aarhus University, Arctic Research Centre (ARC), Denmark \\ *Corresponding Author: Andras Sikter, Department of Internal Medicine, Munici- \\ pal Clinic of Szentendre, Hungary. E-mail: andrassikter3@gmail.com
}

Received: June 15, 2021

Published: July 14, 2021

(C) All rights are reserved by Andras Sikter and Christian Sonne.

\begin{abstract}
Most details of primary hypertension are known, however, it is still unclear how and why the increased activity of angiotensin II and aldosterone cause developing primary hypertension seen worldwide. Here we hypothesize that primary hypertension is an age-related disease initiated by the acidification of the intracellular milieu. The renin-angiotensin-aldosterone system (RAAS), like many other hormones, work through feedback mechanisms, with aldosterone restoring $\mathrm{pH}$ and electrolyte changes produced by respiratory acidosis in targeted cardiovascular and kidney cells. High $\mathrm{NaCl}$ intake enhances the effect of aldosterone (salt-sensitive hypertension). By contrast, angiotensin II restores $\mathrm{H}^{+}$and electrolyte abnormalities caused by metabolic acidosis in the targeted cells (salt-resistant hypertension). Aldosterone and angiotensin II decrease $\mathrm{H}^{+}$concentration synergistically, but act antagonistically on $\mathrm{Cl}^{-}$to $\mathrm{HCO}_{3}{ }_{3}^{-}$concentrations. However, both hormones overcompensate acidosis and increase $\mathrm{Na}^{+}$retention leading to increased circulatory resistance, hypertension, and left-heart hypertrophy by a cascade of events. If this hypothesis is true, the cause of essential hypertension is chronic respiratory and metabolic acidosis and RAAS activation. Preventing age-related intracellular acidosis through a non-hormonal way could prevent pathophysiological blood pressure elevation. It is a challenge to mitigate $\mathrm{pCO}_{2}$ level permanently and we, therefore, recommend a salt mixture of $\mathrm{KH}_{2} \mathrm{PO}_{4}$ and other $\mathrm{Mg}^{2+}, \mathrm{K}^{+}, \mathrm{Ca}^{2+}$ and phosphate salts to relieve the withdrawal symptoms in hypercapnia.
\end{abstract}

Keywords: Intracellular Acidosis; RAAS as a Feedback Mechanism; Salt-resistant Hypertension; Salt-Sensitive Hypertension; Weaning from Hypercapnia

\section{Abbreviations}

COPD: Chronic Obstructive Pulmonary Diseases; CPAP: Continuous Positive Airway Pressure; EBS: Exhausting Buffer Syndrome; EC: Extracellular; IC: Intracellular; NBCn1: Natrium-Bicarbonate Cotranspoter n1; NHE-1: Natrium-Hydrogen Exchanger-1; RAAS: Renin-Angiotensin-Aldosterone System; VSMCs: Vascular Smooth Muscle Cells

\section{Introduction}

It is generally accepted that overproduction and increased activity of the renin-angiotensin system and aldosterone are the direct cause of primary hypertension. Aldosterone is part of the renin-angiotensin-aldosterone system (RAAS), but partly independently enhances sodium chloride sensitivity of hypertension [1]. It is also clear that the effect of excessive table salt consumption on hypertension is primarily related to its chloride content, which is connected to $\mathrm{Na}^{+}[2]$. Both angiotensin and aldosterone concentrations increase during metabolic acidosis giving feedback and trying to neutralize intracellular acidosis on their targeted cells, e.g. in vascular smooth muscle cells (VSMCs) [3,4]. In addition, the effects of angiotensin increases due to low intracellular $\mathrm{HCO}_{3}{ }^{-}$concentration, while elevated cytosolic $\mathrm{HCO}_{3}{ }^{-}$concentration 
increases the aldosterone and sodium chloride effects on the targeted cells [1].

Most of the details of how RAAS causes ionic changes to alkalinize the intracellular milieu are known, showing increased $\mathrm{Na}^{+}$ and $\mathrm{Ca}^{2+}$ concentrations in vascular smooth muscle and myocardial cells, leading to hypertrophy and increased systemic vascular resistance [1]. The causes and pathogenesis of secondary hypertension are well known or elucidated by thorough clinical investigation [5]. By contrast, despite intensive research, the pathophysiologic mechanism of primary hypertension remains unclear, which is why it is also called essential [6]. Recently, it was shown that metabolic acidosis might elicit blood pressure elevation via upregulation of intra-renal angiotensin, being a co-factor in the development of primary hypertension [7]. Primary hypertension is associated with impaired acid-base metabolism, and there may be an association between hypoventilation elicited by chronic psychic stress, persistent $\mathrm{pCO}_{2}$ elevations, and salt-sensitive hypertension $[8,9]$. However, in the case of hypertension, acidosis is overcompensated in the VSMCs. It seems the regulation tries to compensate for all ionic deviations (e.g. also of $\mathrm{Cl}^{-}$and $\mathrm{HCO}_{3}{ }^{-}$alterations) - meanwhile, increasingly severe ion- and consequently causes metabolic disturbance being the ripple effect. The goal can only be the restoration of the original intracellular ionic surroundings and $\mathrm{pH}$, i.e. the elimination of intracellular acidosis.

Here we suggest a speculation about the aetiology of the cardiometabolic syndrome and our newly proposed exhausting buffer syndrome (EBS), defined as the chronic systemic intracellular acidification due to fixed acids' retention. We also assume that intracellular acidosis, caused by a number of different factors such as chronic kidney disease, obstructive sleep apnoea, or acidic diets might be the basis of vascular calcification and primary hypertension [10].

\section{Salt-sensitive hypertension}

Salt-sensitive hypertension is defined as an increase in blood pressure of $10 \%$ or greater due to a diet rich in salt, which, in the presence of aldosterone, increases the $\mathrm{NaCl}$ content in the target cells and increases blood pressure [11]. In salt-resistant hypertension, the aldosterone concentration is reduced during the high salt diet, and blood pressure does not rise. By contrast, in low-renin hypertension, aldosterone level decreases, but the aldostero- ne-to-renin ratio and blood pressure increase (relative aldosterone excess) [12]. A number of inherited salt-sensitive hypertension pathways are linked to sympathetic activations [13-15] while we here discuss acquired salt-sensitive hypertension due to parasympathetic activation [16]. Physiologically, renal blood flow increases when dietary sodium chloride intake increases. In the patients who are having salt-sensitive hypertension, no changes are observed in renal circulation, and a part of the excessive $\mathrm{NaCl}$ was retained [17]. For comparison, relatively mild hypercapnia decreases renal arterial flow; and in COPD, hypercapnia is characterized by hormonal disturbance and salt retention, where the aldosterone level does not diminish either during salt retention $[18,19]$. Hypercapnia induces catabolic effects by causing changes in the tertiary structure of cellular proteins [20]. The assessment of systematic pathophysiological effects of chronic mild hypercapnia may prove that this chronic mild hypercapnia is precisely the biochemical force that triggers cardiometabolic syndrome, including - among other things - type 2 diabetes, salt-sensitive hypertension, vascular calcification and depression [21]. Obstructive sleep apnoea, cardiometabolic syndrome, salt-sensitive hypertension correlate and overlap, and this inhibited breathing and chronic low-grade hypercapnia lead to intracellular acidosis, which is the hypothesized leading cause of cardiometabolic syndrome $[9,10,22,23]$.

We believe that the physiological range of arterial $\mathrm{pCO}_{2}$ is poorly defined (35 - $45 \mathrm{mmHg}$ ) and recommend 38 - $42 \mathrm{mmHg}$ for the normal range of $\mathrm{pCO}_{2}$ levels under 50 years, currently accepted by the minority [24]. Others argue that the upper limit of normal arterial $\mathrm{pCO}_{2}$ should gradually decrease with age due to intracellular acidification caused by the inevitable deterioration of renal function resulting in the exhausting buffer syndrome $[25,26]$. That is, we think that both obstructive sleep apnoea and obesity hypoventilation increase $\mathrm{pCO}_{2}$ to pathophysiological level. Salt-sensitive hypertension is much more frequent in African - Americans than white Americans, likely due to genetic differences [27]. The elevation of $\mathrm{pCO}_{2}$ correlated with the blood pressure sensitivity to high sodium intake and the prevalence of primary hypertension in African Americans above 50 years of age [27]. Nevertheless, Anderson's behavioural chronic stress mechanism may also explain the high prevalence of salt-sensitive hypertension in the African American population that may be partially acquired [28]. We agree with Anderson that persistent, mild hypercapnia may cause acquired salt-sensitive hypertension $[9,10]$. The intracellular $\mathrm{pH}$ chan- 
ges due to hypercapnia have a primary role, so the $\mathrm{pH}$ regulation takes precedence over other ions. A high intake of $\mathrm{NaCl}$ promotes alkalization through the sodium-hydrogen exchanger (NHE-1) and $\mathrm{NaCl}$ entry to these VSMCs by aldosterone [17,29,30]. Aldosterone acts on the NHE-1, alkalizes the VSMCs cytosol and increases their $\mathrm{Na}^{+}$content, triggering events of cascades leading to hypertrophy, increased vascular resistance and blood pressure elevation. Effects of aldosterone and a high intake of $\mathrm{NaCl}$ synergistically support each other. $\mathrm{NaCl}$, more specifically the extracellular-to-intracellular (EC/IC) $\mathrm{Cl}^{-}$ratio, plays a significant role in regulating salt-sensitive hypertension. The salt sensitivity depends on the EC/IC chloride gradient, which is increased in hypercapnic acidosis and decreased in metabolic acidosis [31]. It would explain the correlation why chronic hypercapnia would cause sodium-chloride sensitive hypertension while salt-resistant hypertension remains in metabolic acidosis. As the bicarbonate level changes, the chloride level changes inversely; i.e. in chronic hypercapnic acidosis, the cytosolic $\mathrm{Cl}^{-}$concentration decreases; in metabolic acidosis, it increases [32].

\section{Salt-resistant hypertension}

It was suggested decades ago that intracellular acidosis plays an essential role in the pathophysiology of hypertension (and also diabetes) [33]. Since then, the research in acid-base equilibrium has increased [8], and it is now known that acidosis increases both aldosterone and angiotensin II levels [3,4]. Recently, it was shown that metabolic acidosis could trigger blood pressure elevation in an animal model; thus, it is likely a pathophysiological factor in the development of primary hypertension [7]. In the previous section, we discussed how chronic mild hypercapnic acidosis could trigger acquired salt-sensitive hypertension, while we here demonstrate a link between metabolic acidosis, angiotensin II production, and salt-resistant hypertension. The transition between salt-sensitive and -resistant hypertension is not sharp, because both aldosterone and angiotensin II effects are strongly related (RAAS) and cardiometabolic syndrome and exhausting buffer syndrome are also overlapping [10]. Intracellular ions, such as $\mathrm{Cl}^{-}$and $\mathrm{HCO}_{3}^{-}$levels, as well as pH play a decisive role in regulation [31-34]. Here we discuss changes in RAAS protagonists due to alterations in intracellular ionic milieu, which are feedback mechanisms that try to restore the original intracellular electrolytes.

The most important membrane receptor of angiotensin II is the $\mathrm{Na}^{+} / \mathrm{HCO}_{3}{ }^{-}$cotransporter $\mathrm{NBCn} 1$, and its most essential target cells
- similarly to NHE-1 - are VSMCs and myocardial cells [1]. There are many similarities between the effects of NHE-1 and NBCn1 isoforms. Both occur in the membrane of VSMCs important for the regulation of hypertension, decrease $\mathrm{H}^{+}$and increase $\mathrm{Na}^{+}$concentrations in targeted cells and increase $\mathrm{Na}^{+} / \mathrm{Ca}^{2+}$ exchange and contractility [1]. However, NHE-1 increases intracellular $\mathrm{Cl}^{-}$concentration in the targeted cells; by contrast, $\mathrm{NBCn} 1$ decreases it, as $\mathrm{Cl}^{-}$and $\mathrm{HCO}_{3}{ }^{-}$concentrations are inversely related. Both aldosterone and angiotensin II also regulate the acid-base balance through renal functioning. Both increases renal acid secretion, but aldosterone also increases the $\mathrm{Cl}^{-}$reabsorption [34]. In contrast, angiotensin II is one of the most potent hormonal stimulators of renal $\mathrm{HCO}_{3}^{-}$reabsorption, and acid-base regulation by aldosterone or angiotensin differs [34]. Aldosterone seeks to eliminate respiratory acidosis, while angiotensin II seeks to eliminate metabolic acidosis and its consequences [35]. We hypothesize salt-resistant hypertension would be related to intracellular metabolic acidosis, angiotensin II and NCBn1.

Most chronic kidney diseases are usually characterized by progressive deterioration and secondary hypertension [36] and severe, untreated hypertension leads to chronic kidney damage [37]. We believe that age-related decrease in renal functioning is also inducing intracellular metabolic acidosis, which triggers a compensatory release of renal paracrine hormones such as angiotensin II, aldosterone and endothelin-1 [38]. Intracellular metabolic acidosis is both the cause and consequence of the deterioration, while age-related intracellular metabolic acidosis due to decreased kidney functioning may enhance the intracellular acidosis, and this vicious circle may stop during the elimination of acidosis.

Altogether, this suggests that both metabolic and hypercapnic acidosis increase intracellular $\mathrm{H}^{+}$concentrations increasing, 50+ years of age. These two mechanisms create different syndromes and clinical images. The former is the ensemble defined by the authors as exhausting buffer syndrome, the latter is the cardiometabolic syndrome. The former is salt-resistant, and the latter exerts salt-sensitive hypertension. Due to the overlap, mechanisms are difficult to separate. Concerning intracellular ionic conditions, both lower the intracellular $\mathrm{pH}$, while they behave oppositely regarding intracellular $\mathrm{Cl}^{-}$to $\mathrm{HCO}_{3}{ }^{-}$ratio and $\mathrm{pCO}_{2}$ levels. The two types of acidosis can be brought to a common denominator by compensating for the decrease in intracellular $\mathrm{pH}$ above 50 years by reducing 
$\mathrm{pCO}_{2}$ levels by about 1 - $3 \mathrm{mmHg}[25,26]$. The reduction of metabolic acidosis with $\mathrm{HCO}_{3}{ }^{-}$therapy can only be used to a limited extent (see testing section).

\section{Testing the hypothesis}

The number of researchers who consider intracellular metabolic acidosis to be the leading cause of many age-related diseases is growing. The chronic kidney disease is regarded as a human model of accelerated ageing. The number of studies using sodium bicarbonate therapy as a general solution of metabolic acidosis also increases $[39,40]$. It appears that bicarbonate therapy may be effective even in the prevention of vascular calcification [41], but the $\mathrm{HCO}_{3}{ }^{-}$administration should be limited, because it generates an adverse effect increasing in $\mathrm{pCO}_{2}$ levels, thus triggering intracellular respiratory acidosis [42]. A strategy for restoring mixed intracellular acidosis due to respiratory and metabolic acidosis can be that we should reduce $\mathrm{pCO}_{2}$ levels to slightly below $40 \mathrm{mmHg}[25,26]$.

It is usually hard to change the breathing habit or decrease $\mathrm{pCO}_{2}$ levels, because the breathing regulation fixes it, and the individual's breathing becomes a habit. It is a well-known phenomenon that when the $\mathrm{pCO}_{2}$ level is reduced (e.g. in the case of COPD) by a breathing machine, in the end, it is difficult to wean from the respirator. We are faced with a similar phenomenon if we want to intentionalyly change the respiration frequency or depth, thus reduce the $\mathrm{pCO}_{2}$ level. (This breathing training is recently referred to as „breathing biofeedback"). The success of testing depends on whether we can effectively and permanently increase the respiration minute volume. Therefore, all sorts of tricks need to be figured out.

Theoretically, several methods are possible as outlined below:

First, severe nocturnal obstructive sleep apnea is now routinely treated with continuous positive airway pressure (CPAP) therapy. It has been shown that CPAP treatment in obstructive sleep apnea significantly reduces serum aldosterone and angiotensin II levels as well as blood pressure. However, this may be a consequence of the reduction of hypoxia or hypercapnia [43,44]. Therefore, we suggest lowering the average $\mathrm{pCO}_{2}$ below $40 \mathrm{mmHg}$ by CPAP.

Secondly, the biofeedback of breathing, a computer-controlled breathing training, is based on voluntary alteration of respiration, affecting the $\mathrm{pCO}_{2}$ level. A new breathing training method using capnography appears to be suitable for a permanent mild reduction of the $\mathrm{pCO}_{2}$ level [45].
Thirdly, intentional therapeutic alteration of the $\mathrm{pCO}_{2}$ level moves the patient from a stable position to a labile one. Decreased $\mathrm{pCO}_{2}$ levels result in a reduction in intracellular acidosis, a sudden onset of cellular anabolic metabolism, and essentially the development of the refeeding syndrome [46]. Severe hypophosphatemia occurs when the $\mathrm{pCO}_{2}$ level is decreasing, and it is a significant cause of the "difficult-to-wean" phenomenon [47], usually accompanied by hypomagnesaemia and hypokalemia [48]. There is a inevitable loss in bone mass during acidosis [25], however, after $\mathrm{pH}$ restoring, the tissue rebuild phosphate and $\mathrm{Ca}^{2+}$, resulting in hypocalcemia and hypophosphatemia (hungry bone syndrome) [49].

We believe and know that there should be a salt mixture that not only restores the electrolyte deficiency caused by decrease in $\mathrm{pCO}_{2}$ but, at the same time, also decreases $\mathrm{pCO}_{2}$ levels [50]. Its composition may vary slightly from individual to individual, but its essential constituent is $\mathrm{KH}_{2} \mathrm{PO}_{4}$, which increases breathing and decreases $\mathrm{pCO}_{2}$ level. This call for a $\mathrm{K}_{2} \mathrm{HPO}_{4}$ supplement; the ratio of $\mathrm{KH}_{2} \mathrm{PO}_{4} / \mathrm{K}_{2} \mathrm{HPO}_{4}$ - according to this testing hypothesis - is able to regulate the breathing and $\mathrm{pCO}_{2}$ levels. If the ratio of two phosphate salts administered increases, the $\mathrm{pCO}_{2}$ will decrease and vice versa. The amount to be administered should be titrated individually based on laboratory findings monitoring. It is advisable to satisfy with $\mathrm{Mg}^{2+}, \mathrm{Ca}^{2+}, \mathrm{Zn}^{2+}$, other ions, trace elements and vitamins, as described for refeeding and hungry bone syndromes. Therefore, we call for treatment, including that physiological salt mixture that reduces mild hypercapnia and improves metabolism and lowers high blood pressure.

\section{Conclusion}

Sufficient literature data is available to puzzle out the main features of the pathophysiology of age-related or civilization diseases, such as primary hypertension. The signs of intracellular acidosis already manifest in more than $90 \%$ of people over 50 years. In most cases, metabolic acidosis and respiratory acidosis co-occur; where one predominates, the other does not, thus they overlap. Chronic kidney disease (as an example of metabolic acidosis) and obstructive sleep apnea (as a respiratory acidosis) are considered human models for accelerated ageing. The former manifests itself primarily in the form of exhausting buffer syndrome, the latter that of cardiometabolic syndrome. In both, there is intracellular acidosis, but the cytosolic $\mathrm{Cl}^{-} / \mathrm{HCO}_{3}{ }^{-}$ratio is higher in EBS than in cardiometabolic syndrome. Regarding primary hypertension: in metabolic acidosis, angiotensin II triggers salt-resistant hypertension in 
VSMCs via NBCn1 receptors, while in chronic respiratory acidosis, aldosterone and high $\mathrm{NaCl}$ levels together trigger salt-sensitive hypertension through NHE-1.

Increases in both aldosterone and angiotensin II levels can be initiated by intracellular $\mathrm{H}^{+}[3,4]$. If this statement is confirmed and the process can be stopped by intracellular alkalinization, on the other hand, the "essential" name can no longer be used. This hypothesis points not only to the paramount importance of intracellular $\mathrm{pH}$ and the extreme $\mathrm{pH}$ sensitivity of proteins, but also to the fact that ions considered to be insignificant such as $\mathrm{Cl}^{-}$do have metabolism regulatory roles, and the intracellular ion milieu as a whole is an integral part of the second messenger system [46].

Like many other hormonal systems, RAAS functions as a feedback system that seeks to restore intracellular $\mathrm{pH}$. Still, its activity is mainly limited to VSMCs, myocardial and certain kidney cells, serving only a relatively small slice of the body's tissue regulation. It has pathophysiological effects, because the impact in the target cells exceeds the target.

What to do? The testing menu suggested by the authors is easy to check; the result determines everything.

\section{Acknowledgements}

The authors would like to thank Ms Krisztina Toth for proofreading the manuscript.

\section{Bibliography}

1. Rosskopf D., et al. "Membrane sodium-proton exchange and primary hypertension”. Hypertension. 21.5 (1993): 607-617.

2. Chipperfield AB and Harper AA. "Chloride is smooth muscle". Progress in Biophysics and Molecular Biology 74.3-5 (2000): 175-221.

3. Ng HY., et al. "Activation of intrarenal renin-angiotensin system during metabolic acidosis". American Journal of Nephrology 34.1 (2011): 55-63.

4. Wesson DE., et al. "Acid retention accompanies reduced GFR in humans and increases plasma levels of endothelin and aldosterone". American Journal of Physiology - Renal Physiology 300.4 (2011): F830-837.
5. Charles L., et al. "Secondary Hypertension: Discovering the Underlying Cause". American Family Physician 96.7 (2017): 453-461.

6. Carretero OA and Oparil S. "Essential hypertension. Part I: definition and etiology". Circulation 101.3 (2000): 329-335.

7. Aryal D., et al. "Chronic metabolic acidosis elicits hypertension via upregulation of intrarenal angiotensin II and induction of oxidative stress". Antioxidants Basel 10.1 (2021): 2. doi: 10.3390/antiox10010002.

8. Boedtkjer E and Aalkjaer C. "Disturbed acid-base transport: an emerging cause of hypertension". Frontiers in Physiology 4 (2013): 388. doi: 10.3389/fphys.2013.00388

9. Anderson DE. "Cardiorenal effects of behavioral inhibition of breathing". Biological Psychology 49.1-2 (1998): 151-163.

10. Sikter A and Sonne C. "A new hypothesis on vascular calcification: the exhausting buffer syndrome (EBS)". Neuropsychopharmacology Hung 23.1 (2021): 215-220.

11. Choi HY., et al. "Salt sensitivity and hypertension: a paradigm shift from kidney malfunction to vascular endothelial dysfunction". Electrolyte Blood Press 13.1 (2015): 7-16.

12. Satoh M., et al. "Aldosterone-to-renin ratio and nocturnal blood pressure decline assessed by self-measurement of blood pressure at home: the Ohasama Study". Clinical and Experimental Hypertension 36.2 (2014): 108-114.

13. Fujita T. "Mechanism of salt-sensitive hypertension: focus on adrenal and sympathetic nervous systems". Journal of the American Society of Nephrology 25.6 (2014): 1148-1155.

14. Snyder EM., et al. "Arg16Gly polymorphism of the beta2-adrenergic receptor is associated with differences in cardiovascular function at rest and during exercise in humans". Journal of Physiology 571.1 (2006): 121-130.

15. Pojoga L., et al. "Beta- 2 adrenergic receptor diplotype defines a subset of salt-sensitive hypertension". Hypertension 8.5 (2006): 892-900.

16. Anderson DE., et al. "Salt sensitivity of blood pressure is accompanied by slow respiratory rate: results of a clinical feeding study". Journal of the American Society of Hypertension 1.4 (2007): 256-263. 
17. Williams GH and Hollenberg NK. "Non-modulating hypertension. A subset of sodium-sensitive hypertension". Hypertension 17.1 (1991): I81-185.

18. Chapman CL., et al. "Renal and segmental artery hemodynamic response to acute, mild hypercapnia". American Journal of Physiology - Regulatory, Integrative and Comparative Physiology 318.4 (2020): 822-R827.

19. Valli G., et al. "Water and sodium imbalance in COPD patients". Monaldi Archives for Chest Disease 61.2 (2004): 112-116.

20. Relman AS. "Metabolic consequences of acid-base disorders". Kidney International 1 (1972): 347-359.

21. Balnis J., et al. "Hypercapnia-Driven Skeletal Muscle Dysfunction in an Animal Model of Pulmonary Emphysema Suggests a Complex Phenotype". Frontiers in Physiology 11 (2020): 600290. doi: 10.3389/fphys.2020.600290.

22. Rocchini AP. "Obesity hypertension, salt sensitivity and insulin resistance". Nutrition, Metabolism and Cardiovascular Diseases 10.5 (2000): 287-294.

23. Pimenta E., et al. "Increased dietary sodium is related to severity of obstructive sleep apnea in patients with resistant hypertension and hyperaldosteronism". Chest 143.4 (2013): 978-983.

24. Bruton A and Holgate ST. "Hypocapnia and asthma. A mechanism for breathing retraining?” Chest 127 (2005): 1808-1811.

25. Frassetto L and Sebastian A. "Age and systemic acid-base equilibrium: analysis of published data". Journals of Gerontology Series A Biological Sciences and Medical Sciences 51.1 (1996): B91-99.

26. Dhokalia A., et al. "Resting end-tidal CO2 association with age, gender, and personality". Psychosomatic Medicine 60.1 (1998): 33-37.

27. Anderson DE., et al. "Racial differences in resting end-tidal $\mathrm{CO}_{2}$ and circulating sodium pump inhibitor". American Journal of Hypertension 14.8 (2001): 761-767.

28. Anderson DE. "Respiratory psychophysiology in hypertension research". Behavior modification 25.4 (2001): 606-620.

29. Pimenta E., et al. "Salt, aldosterone and hypertension". Journal of Human Hypertension 27.1 (2013): 1-6.
30. Whaley-Connell A., et al. "Aldosterone: role in the cardiometabolic syndrome and resistant hypertension". Progress in Cardiovascular Diseases 52.5 (2010): 401-409.

31. Chipperfield AB, and Harper AA. "Chloride is smooth muscle". Progress in Biophysics and Molecular Biology 74.3-5 (2000): 175-221.

32. Feldman M and Dickson B. "Adjusted Plasma Chloride and Bicarbonate Concentrations: An Approach to Identifying AcidBase Disorders". Journal of Applied Laboratory Medicine 2.6 (2018): 833-840.

33. Resnick LM., et al. "Intracellular $\mathrm{pH}$ in human and experimental hypertension". Proceedings of the National Academy of Sciences of the United States of America 84.21 (1987): 7663-7667.

34. Wagner CA. "Effect of mineralocorticoids on acid-base balance". Nephron Physiology 128.1-2 (2014): 26-34.

35. Zhou Y., et al. "Effects of angiotensin II on the CO2 dependence of HCO3- reabsorption by the rabbit S2 renal proximal tubule". American Journal of Physiology - Renal Physiology 290.3 (2006): F666-673.

36. Fogo AB. "Mechanisms of progression of chronic kidney disease". Pediatric Nephrology 22.12 (2007): 2011-2022.

37. Bidani AK and Griffin KA. "Pathophysiology of hypertensive renal damage: implications for therapy". Hypertension 44.5 (2004): 595-601.

38. Wesson DE., et al. "Mechanisms of Metabolic Acidosis-Induced Kidney Injury in Chronic Kidney Disease". Journal of the American Society of Nephrology 31.3 (2020): 469-482.

39. Wesson DE. “Is NaHCO3 an antiaging elixir?" American Journal of Physiology - Renal Physiology 311.1 (2016): F182-F183.

40. Melamed ML and Raphael KL. "Metabolic Acidosis in CKD: A Review of Recent Findings”. Kidney Medicine 3.2 (2021): 267277.

41. Leibrock CB., et al. "Bicarbonate-sensitive calcification and lifespan of klotho-deficient mice". American Journal of Physiology - Renal Physiology 310.1 (2016): F102-F108.

42. Forni LG., et al. "The Janus faces of bicarbonate therapy in the ICU: not sure!" Intensive Care Medicine 46.3 (2020): 522-524. 
43. Nicholl DDM., et al. "Nocturnal hypoxemia severity influences the effect of CPAP therapy on renal renin-angiotensin-aldosterone system activity in humans with obstructive sleep apnea". Sleep 44.5 (2021): zsaa228. doi: 10.1093/sleep/zsaa228.

44. Iftikhar IH., et al. "Effects of continuous positive airway pressure on blood pressure in patients with resistant hypertension and obstructive sleep apnea: a meta-analysis". Journal of $\mathrm{Hy}$ pertens 32.12 (2014): 2341-2350.

45. Szulczewski MT. "Training of paced breathing at $0.1 \mathrm{~Hz}$ improves $\mathrm{CO} 2$ homeostasis and relaxation during a paced breathing task". PLoS One 14.6 (2019): e0218550. doi: 10.1371/journal.pone. 0218550

46. Sikter A. "Psychosomatic Molecular Mechanisms of Metabolic Syndrome and Type 2 Diabetes. Part 1. A Theory for Modelizing the Cytoplasm and Diseases". Acta Scientific Medical Sciences 4.1 (2020): 124-140. doi: 10.31080/ASMS.2019.04.

47. Zhao Y., et al. "Effect of hypophosphatemia on the withdrawal of mechanical ventilation in patients with acute exacerbations of chronic obstructive pulmonary disease". Biomed Report 4.4 (2016): 413-416.

48. Seyyedi J., et al. "Comparison of the Effect of Enteral Feeding through the Bolus and Continuous Methods on Serum Phosphorus and Glucose Levels in Patients with Mechanical Ventilation: A Randomized Clinical Trial". Journal of Nutrition and Metabolism (2020): 6428418. doi: 10.1155/2020/6428418.

49. Frisch LS and Mimouni F. "Hypomagnesemia following correction of metabolic acidosis: a case of hungry bones". Journal of the American College of Nutrition 12.6 (1993): 710-713.

50. Sikter A. "Psychosomatic Molecular Mechanisms of Metabolic Syndrome and Type 2 Diabetes. Part 4. A Recovery Strategy from Chronic Hypercapnia and Metabolic Syndrome-Related Disorders, (a Research Proposal)". Acta Scientific Medical Sciences 4.3 (2020): 150-161. doi:10.31080/ASMS.2020.04.0578

\section{Volume 5 Issue 8 August 2021}

(C) All rights are reserved by Andras Sikter and Christian Sonne.

Citation: Andras Sikter and Christian Sonne. "Is the Primary Aetiology of Hypertension Unknown? Novel Views on Previous Assumptions". Acta Scientific Medical Sciences 5.8 (2021): 47-53. 